\title{
Simulation of Sparse Model for Fmri Signal with Brain Activation During Hunger Regulation Process
}

\section{Divya, Saurabh Mukherjee}

Abstract: Functional Magnetic Resonance Imaging (fMRI), a non-invasive technique, is used for the recognition of different Cerebral Blood Flow (CBF) and Blood Oxygenated level dependent (BOLD) measures which result into the identification of various neural activities related to different physiological processes such as Hunger Regulation, Water Balancing etc. Different BOLD contrast levels (blood oxygenated and deoxygenated level) specify diversity in various state of human brain functioning subject to various tasks. The proposed model is a hybrid combination of Sparse method (Carroll et al., 2009) and Hypothalamic Hunger Regulation Model i.e. Sparse matrix for Hypothalamic BOLD Signal method (SMHB Method). SMHB method is dynamic and linear in nature. It defines the sparse parameters which act on the mapping between the fMRI signal for hunger regulation process and sparse representation of the signal segmented from the input image by which every voxel of fMRI signal in temporal domain can be expressed as a sparse signal. A sparse model provides a well define results for task based localized activity. It can be applied on a single image as well as an FMRI dataset. The implementation of SMHB method divided into different sub-modules such as Input image analysis and visualization, Linear Voxel Module and Neuro Activation Module. Our study have completed first two module with different pre-processing techniques used for image analysis and linear representation of each voxels of fMRI signal in the form of sparse parameters.

Keywords: Hunger Regulation, fMRI, BOLD, Linear Sparse Model, Sparse Matrix.

\section{INTRODUCTION}

The complex technique of fMRI has been studied through different models and numerical simulation. The fMRI signal depends on neurovascular factors, brain activity for physiological and homeostatic functions, oxygen metabolism, neurovascular coupling and more [7]. The Homeostatic functions has been described with mathematical perspective under consideration of various spatial and statistical parameters like fractals, entropy, membership function, wavelets and correlation, variance and Skewness respectively [1][2]. An accurate analysis of BOLD contrast gives correct interpretation of the physiological functions. Different biophysical models calibrated and quantify the functional changes. The basic key features of such methods are mono-variant and multivariant, Linear and Non Linear, convolution, regression and covariance etc.

Revised Manuscript Received on February 05, 2020.

* Correspondence Author

Divya*, Assistant Professor,Department of Computer Science, Banasthali Vidyapith, Rajasthan.

Saurabh Mukherjee, Professor, Department of Computer Science, Banasthali Vidyapith, Rajasthan.

(C) The Authors. Published by Blue Eyes Intelligence Engineering and Sciences Publication (BEIESP). This is an open access article under the CC BY-NC-ND license (http://creativecommons.org/licenses/by-nc-nd/4.0/)
BOLD fMRI simulators and software has been developed under the challenging environment created by these physiological based parameters. The fMRI technique reveals certain level of information regarding the motor, metabolic, cognitive, physiological and perceptual based neural activity of human brain. Such studies required a high resolution data set with variation in tasking [7] [8]. The increase in local CBF results in the increase in the metabolic level of oxygen and glucose. Due to the complexity in BOLD signal an optimized model is required for the vascular, physiological and metabolic interpretation of different brain regions [8]. Different categories for the Analytical Models and Simulation methods for the BOLD signals are introduced with passage of time. Biophysical Simulations methods for BOLD signals single voxel model, multi-voxel model and multi-resolution models [14]. Analytical category based BOLD models are Static and Dynamic Model (Figure 1) Dynamic Model further categorised as Linear and NonLinear Sparse Model, Calibrated Model and Balloon Model. Our study is mainly concentrated on linearly represented Sparse Model.

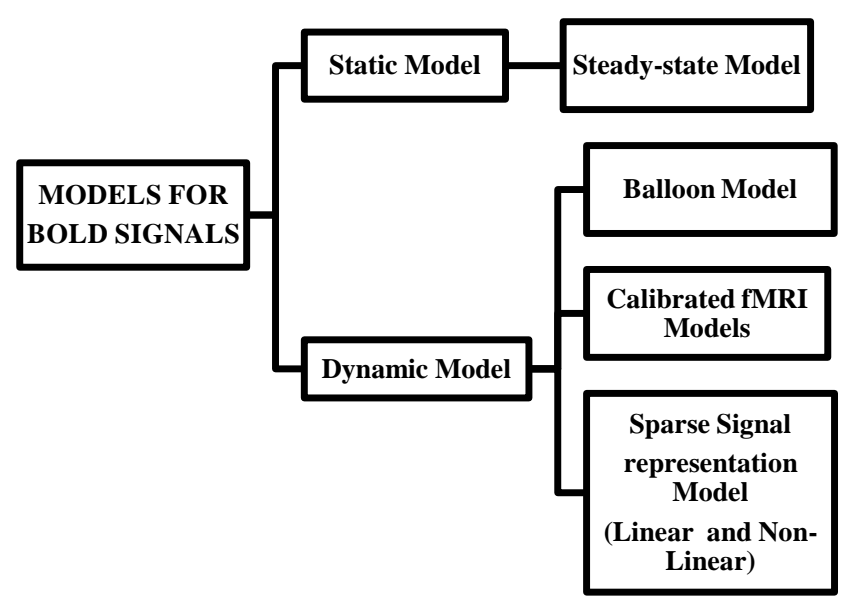

Figure 1: Categories of Models for BOLD signal representation and Analysis

\section{LITERATURE REVIEW}

From last decade a number of models based on different parametric input and functionality has been applied to fMRI data to describe different activity and task based result. BOLD, DWI, MEMRI, HMR spectroscopy based methods provide neuro compartment based interpretation of fMRI signals with metabolic and neuro-endocrine task and functions of human body [15] [16]. 
Pulse Arterial Spin Labelling Magnetic Resonance Imaging (PASL MRI) can also be used to create discrepancy between the signals generated for food deprivation and satiety in healthy obese humans. Satiety hormone i.e. Leptin triggered the neurons at arcuate nucleus at Hypothalamus for increase and decrease in food consumption and energy expenditure [3]. The functionality based connection between Hypothalamus and pituitary gland differentially influence the BMI (Body Mass Index) of human body positively. The multiple linear regression based method has been used for this implementation [5]. Analysis and Classification techniques can be apply on different metabolic functions like hunger regulation, energy balance, water balance etc. A classical method, Machine learning classifier (MLC), can be used to classify different brain activities. Multi-voxel pattern Analysis (MVPA) examines the mapping between activities and cognitive states of brain. Support Vector Machine classifier with machine learning scenario gives more than 80 $\%$ accuracy on fMRI signals with resting state to differentiate between two major metabolic states of Homeostasis process i.e. Hunger and Satiety state[6]. A convolution based model is constructed for the fMRI signals classification for multiple tasks depends on Regression based General Linear Model (GLM) and gives output with variation in BOLD signal with time based parameters [7]. Multivariate methods are more relevant and optimized to map the complexity of the fMRI imaging techniques. An elastic network method interpret fMRI activity network to various task and its parameters [9].A multivariate method using sparse representation can be used positively to analyse various states and groups of brain network for Autism disorder. The Multilink analysis method provides an optimal result with an above average accuracy level defining the cross dependency and connectivity between different features of Brain networks [4]. A different range of methods can also be used to filter noise from the fMRI signal. These noises can be either instrumental because of machine or physiological fluctuations or object movement based noise or many more. For the de-noising process, regression (Global signal regression method) and variance based method can be applied on the acquired fMRI signal [12]. For spatial fMRI dataset with richer texture and with noise Empirical mode decomposition model with green function resultant an optimized outcomes than previously used standard methods but increase the computational weight to the machine [13]. Wavelets transform is beneficial with both spatial domain and temporal domain fMRI signals. The decomposition and de-correlation properties of Wavelets transform provides parametric lead to General Linear Model's variables and the activity map of an fMRI signal. For Sparse Representation, a Threshold based iterative algorithm is proposed for an activity which is sparsely distributed in time [17].

\section{MOTIVATION}

Apart from providing the mapping between the voxel of the fMRI signal and sparse array, gives multiple optimized outcomes which are as follows:

- It gives mapping for the aperiodic activity of any neuron with respect to time individually.
- The response of a single neuron from passive to generating spike for a selective stimulus activity generating by group of neurons.

- For a particular point of time range, the stimulation of a small cluster of neurons to a single stimulus activity.

- Sparse dictionary learning based model can be applied to complete fMRI dataset.

- It determines and interprets the task based activity parameters from an fMRI activity pattern.

- Pattern analysis and classification can be performed with multivariate model.

\section{METHODOLOGY}

Our Simulation method, Sparse Model [9] [10] for Hypothalamic BOLD signal (SMHB) described by the following four steps (Figure 2) including the equations (procedures) and assumptions.

Step 1: Input image is the synthesized image which required multiple methods image analysis to visualize the image features.

Step 2: Each fMRI voxel's element is sparse and the activation spikes integration for that neuron is linear in nature. The observations at $i^{\text {th }}$ voxel stimuli generated by a single neuron i.e. variable $n_{i}$ can be calculated using Linear Voxel Module where the column vector of generated matrix (S) can be map to the fMRI signal for a single neuron.

$$
\sum_{i=1}^{c} n_{i}=S * V_{i}+\varepsilon_{i}
$$

Such that $n_{i}$ belongs to $\mathbb{R}$ with $M$ dimension and where $i$ can be number of voxel such that $\mathrm{i}=1,2 \ldots \mathrm{C}, \mathrm{S}$ is matrix with dimension $\mathrm{M} \times \mathrm{N}$, generated after fMRI scan for a single experimental task. $\mathrm{V}_{\mathrm{i}}$ is the optimized average signal potential with a number of $\mathrm{P}$ parametric functions. $\varepsilon_{i}$ is the noise at each voxel ( $i^{\text {th }}$ voxel). For (Equation 1) each stimulated spike for a single neuron is equivalent to the experimental pattern in the parametric dictionary.

Step 3: Using convolution and Hemodynamic response function two parametric dictionary introduced for our resultant matrix X. For Neuron Activation Module (NAM) for any Sparse representation model a statistical model can be used. To prompt discrepancy and sparseness to our model Laplacian function [9]. Laplacian function gives the statistical mapping between the different stimuli and regression individually applied to each $\mathrm{V}_{\mathrm{i}}$, fMRI signal.

$$
\begin{array}{r}
H_{i}=P^{D} * R\left(V_{i}\right) \\
\text { For } \mathrm{i}=1,2 \ldots \mathrm{C} .
\end{array}
$$

Further calculation includes False Probability Detection Function (FPDF) and Stimulus Threshold Evaluation (STE). 
A threshold variable $\mathbf{T}_{\mathbf{v}}$ for each stimulus is calculated from a Laplacian distribution function.

Step 4: Result will be in the represented in the form of Sparse matrix. Our

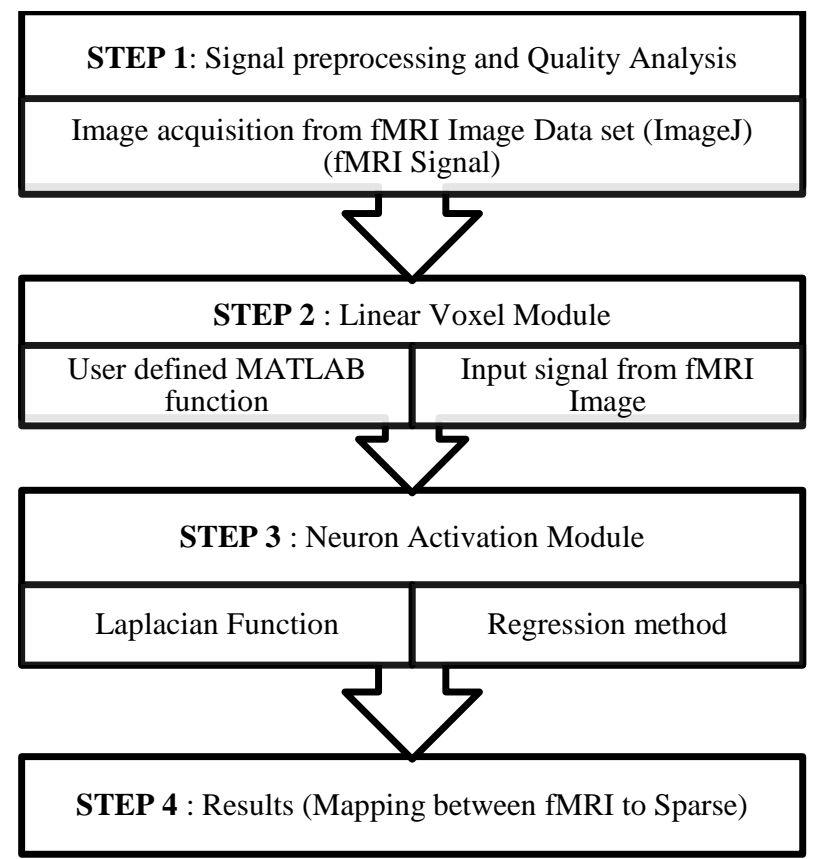

Figure 2: Step by Step procedure for Simulation of Sparse Model for Hypothalamic BOLD signal (SMHB)

\section{V.RESULTS AND DISCUSSION}

The deployment of the SMHB model can be tool-wise divided into two major parts: ImageJ (1.52a, Java1.8.0_112 (64bit)) and MATLAB R2018a. ImageJ is used to acquire the data (Image) specific to neural activity at Hypothalamus for food and non-food images [11]. For our result which include image acquisition and analysis of input data. With help of MATLAB R2018a, a series of user defined functions has been developed for each equation (Equation 1 and 2). The result from our study can be described by the following points:

- Input Image: For pre-processing, an image (32 bits) with resolution 175 X 288 X 288 (length, width, slice) is used for input (Figure 3 and Figure 4).

- Pre-processing method: It includes contrast enhancement and sharpening (Figure 7) and quality analysis for fMRI signal includes histogram
(Figure 5 and Figure 9) and Fourier transforms method (Figure 11(a) and 11(b)).

- Transform function: Fire colormap (Figure 3(c)) with variables colours and alphas, Thermal colormap and surface plot are applied to enhance the visibility of the images.

- Localization (Figure 4): For the location of Hypothalamus in the input image the Co-ordinated value for the voxel $x=87, y=148, z=94$ and voxel value $=79$ and rotation co-ordinates are $\mathrm{x}=86 \mathrm{y}=$ $82 \mathrm{z}=4$.

- Local Thresholding (bitwise figure 8): The methods provided by ImageJ to show active different neuro-compartments of brain at threshold range which are Maxentropy method, Otsu method and Percentile method (figure 6).

Table I: Threshold values for Local Thresholding

\begin{tabular}{|l|l|l|l|}
\hline $\begin{array}{l}\text { Threshold } \\
\text { Level }\end{array}$ & $\begin{array}{l}\text { Max } \\
\text { Entropy }\end{array}$ & $\begin{array}{l}\text { Otsu } \\
\text { method }\end{array}$ & $\begin{array}{l}\text { Percentile } \\
\text { Method }\end{array}$ \\
\hline Minimum & 106 & 98 & 101 \\
\hline Maximum & 125 & 110 & 110 \\
\hline
\end{tabular}

Table II: Histogram Parameters (Figure 9)

\begin{tabular}{|l|l|l|}
\hline Count & Mean & StdDev \\
\hline 480053 & 31.622 & 52.859 \\
\hline Min & Max & Mode \\
\hline 0 & 255 & 0 (301849) \\
\hline
\end{tabular}

- The histogram (Figure 5 and 9) of the input image to represent intensity level (weighted and unweighted) with the histogram parameters like no of pixels, mean and standard deviation shows in Table I and II.

- Contrast, saturation and edges enhanced image is used for further processing where we used the Fast Fourier Transform (figure 11 (a) and 11 (b)) and surface plot (figure 10(a) and (b)) to visualize the voxels' value for the co-ordinated value $(\mathrm{x}, \mathrm{y}, \mathrm{z})$. The Fourier transform of the original image and the edge enhanced image can be easily differentiated visually and parameterized level.

Two modules have been implemented successfully and results have been displayed with the help of Figure 3 to 11 . 

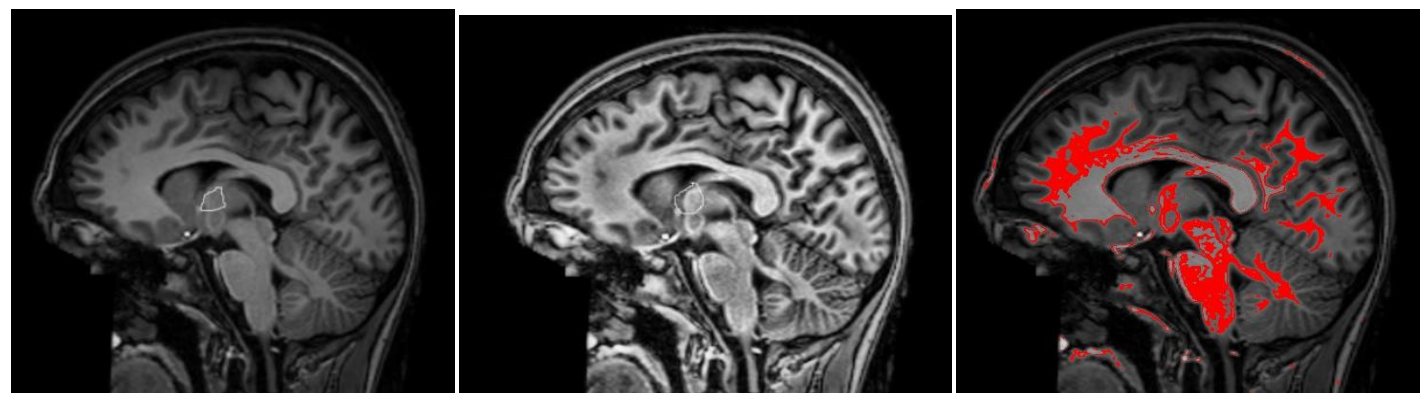

Figure 3: (a, b, c) Location of Hypothalamus in an input Image

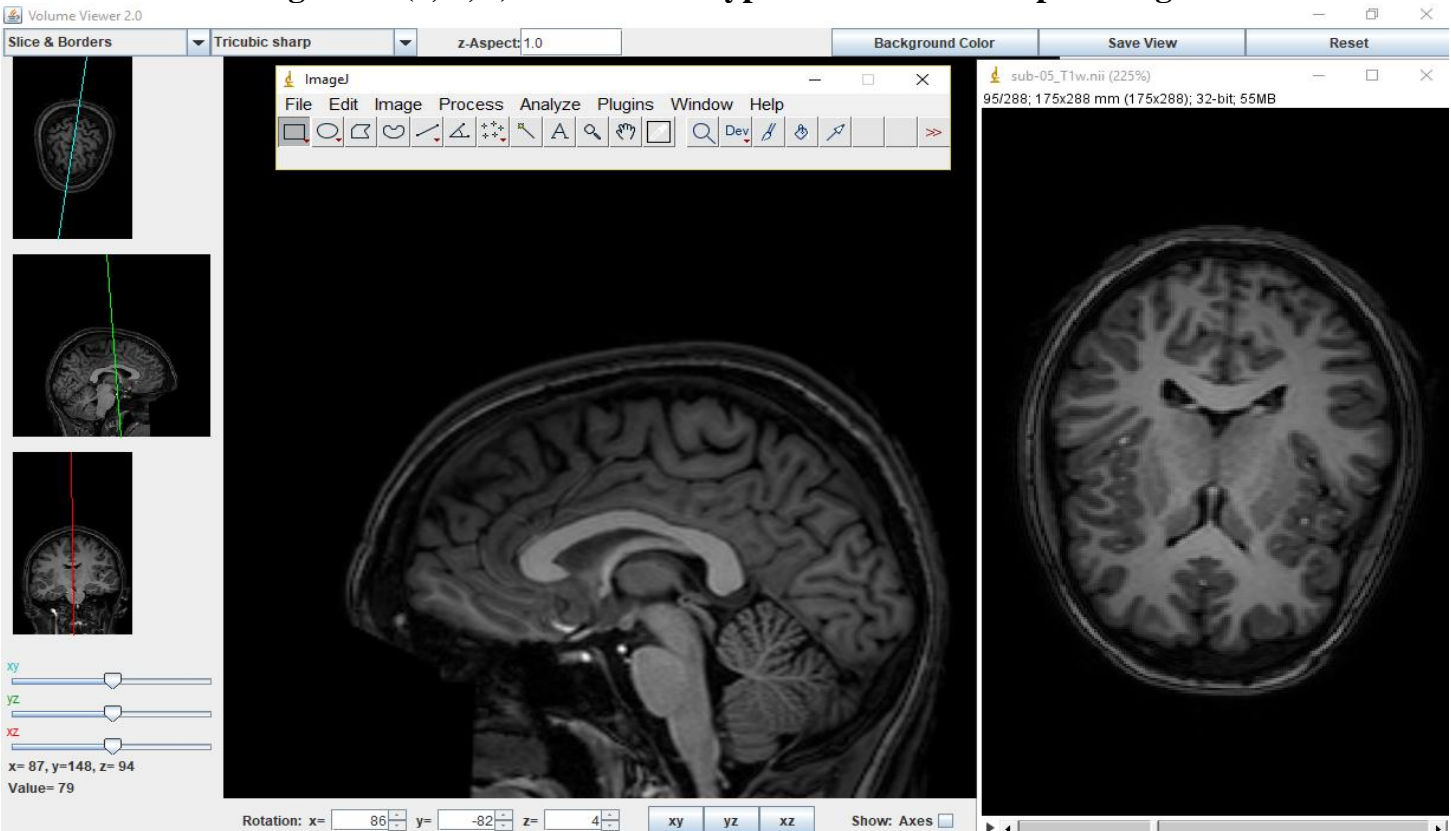

Figure 4 : ImageJ environment used to locate the Hypothalamus in a data set (Sequence of Images (32 bit) with 288 slices) of resolution 175 X 288.
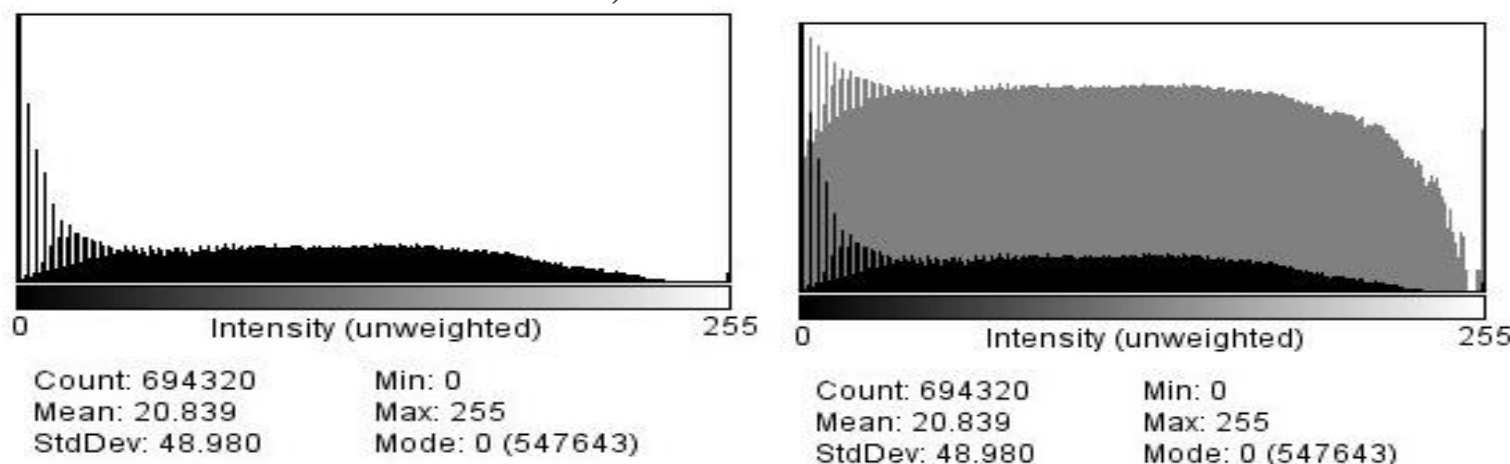

$\begin{array}{ll}\text { Count: } 694320 & \text { Min: } 0 \\ \text { Mean: } 20.839 & \text { Max: } 255 \\ \text { StdDev: } 48.980 & \text { Mode: } 0 \text { (547643) }\end{array}$

Figure 5 : Histogram of the original Image (Figure 4) with un-weighted Intensity and mean and standard Deviation
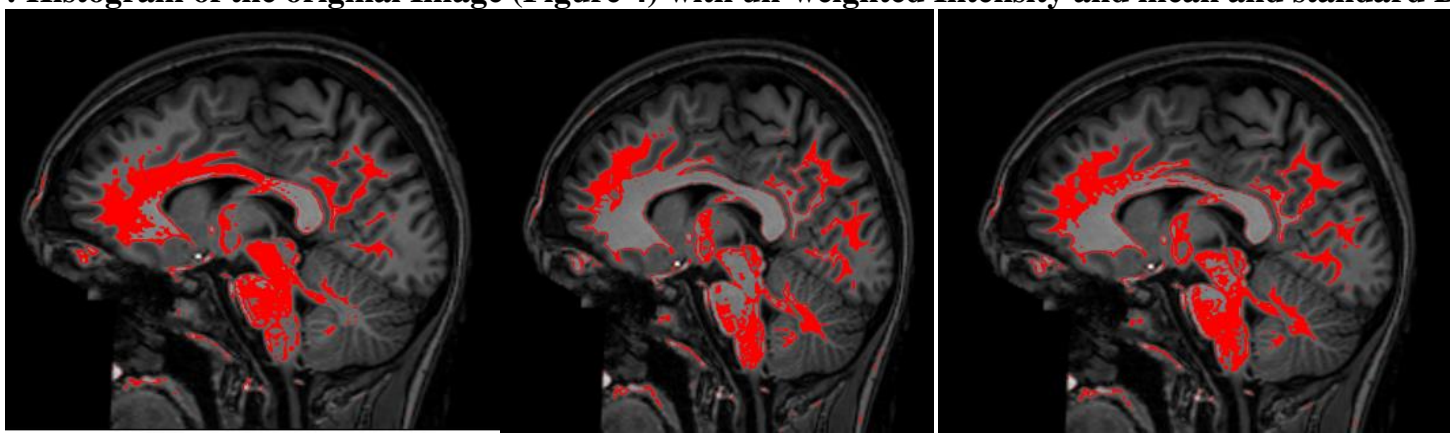

Figure 6 (a, b, c): Resultant Images with MaxEntropy, Otsu method and Percentile method for local Thresholding.

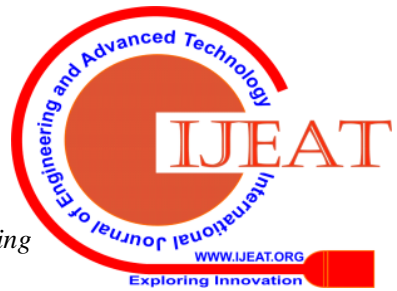




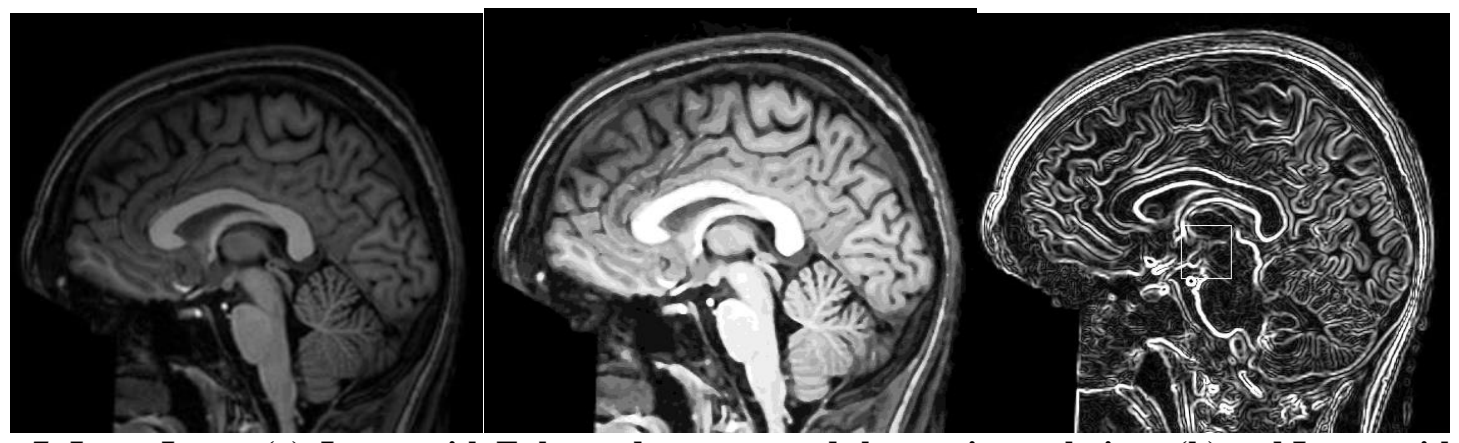

Figure 7: Input Image (a), Image with Enhanced contrast and sharpening technique (b) and Image with edge identification (c).

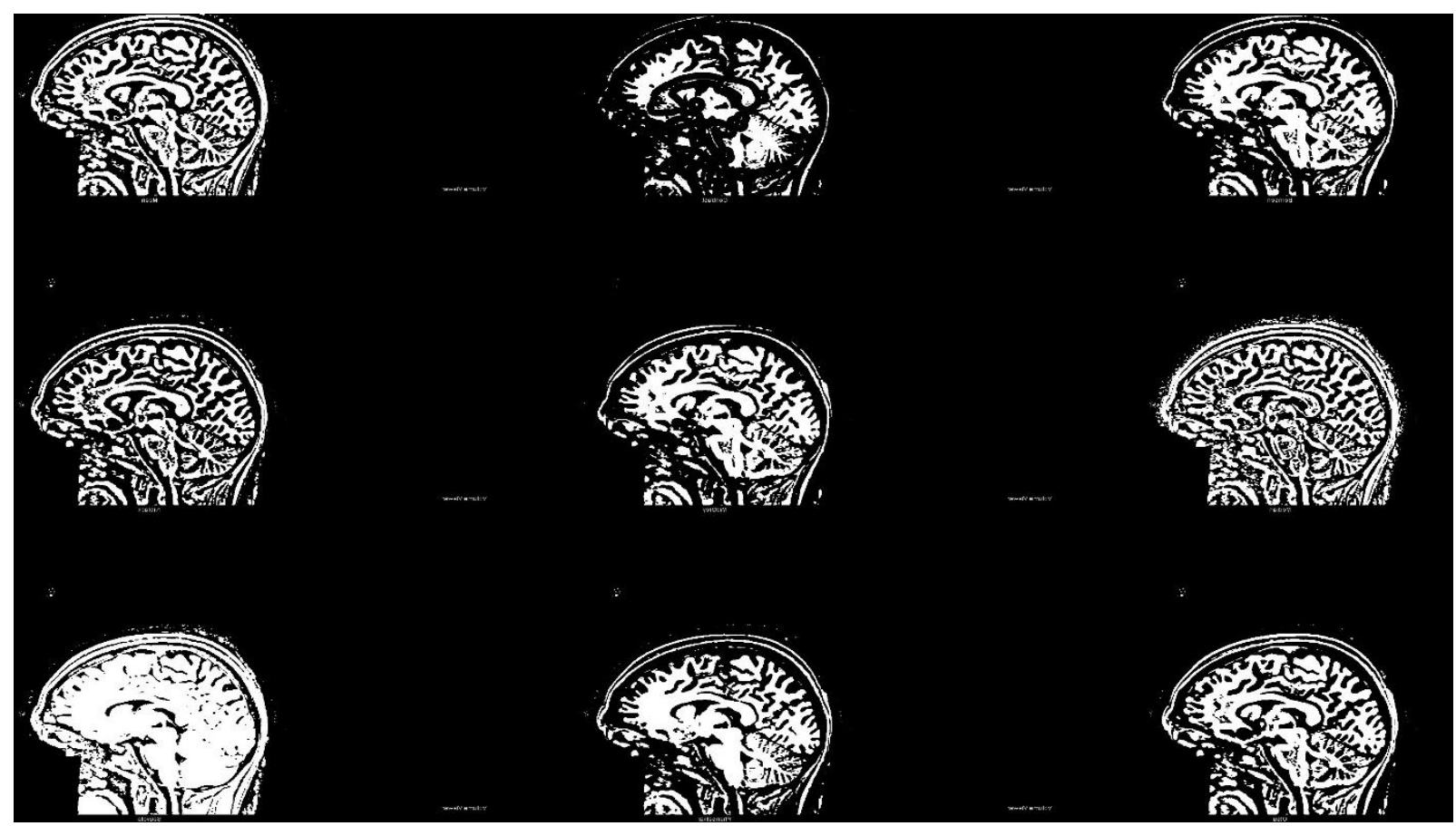

Figure 8 fMRI Image after local threshold method (bitwise) of an 8 bit grayscale image.

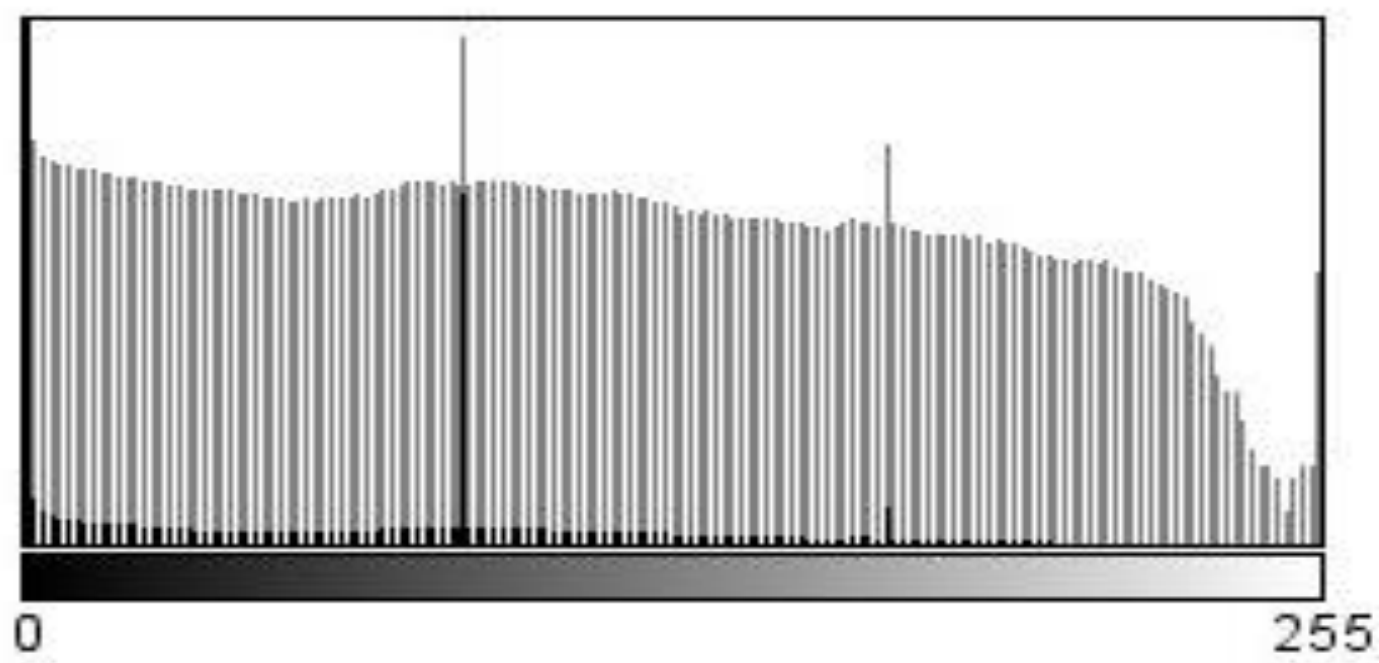

Figure 9: Histogram for image 9(a)

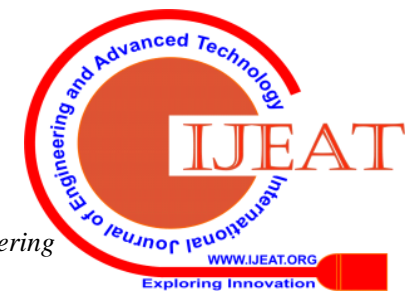



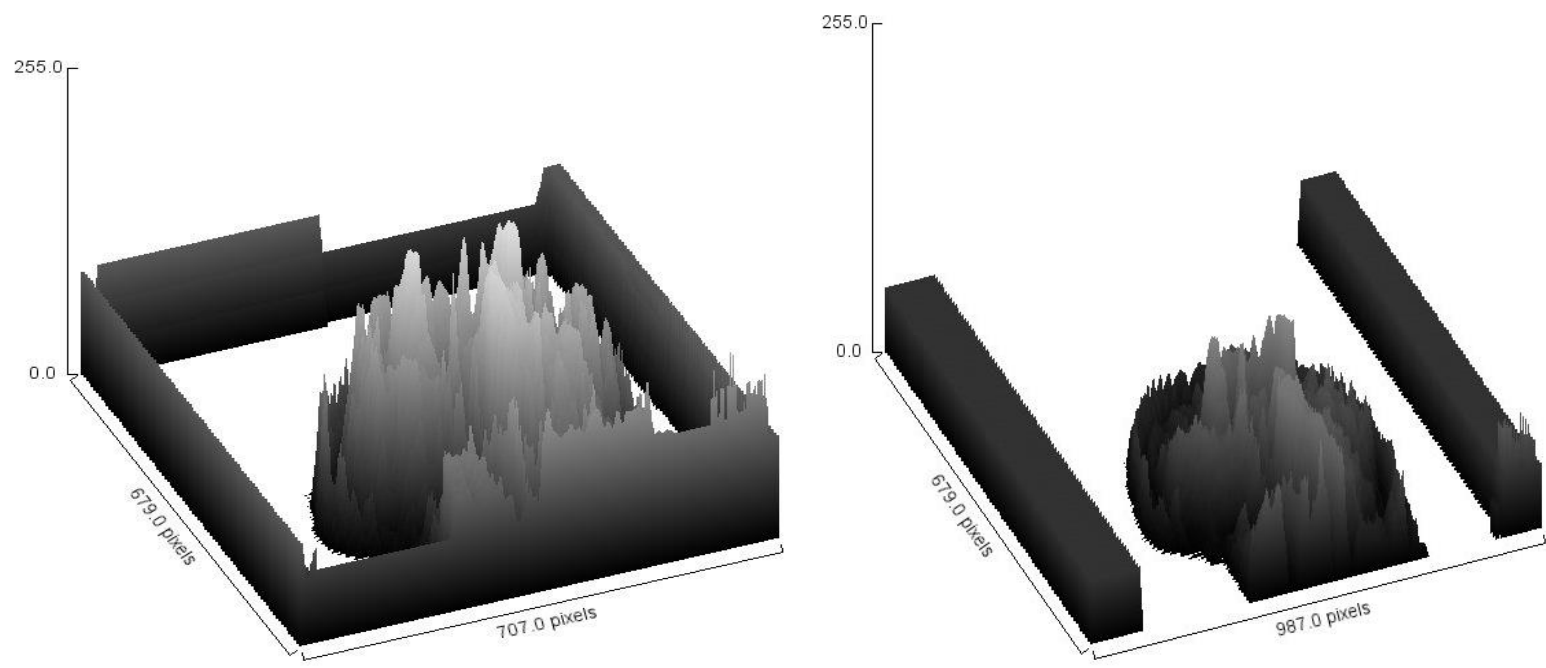

Figure 10 : (a) Surface plot for image 9(a), (b) Surface plot for image 9(b).
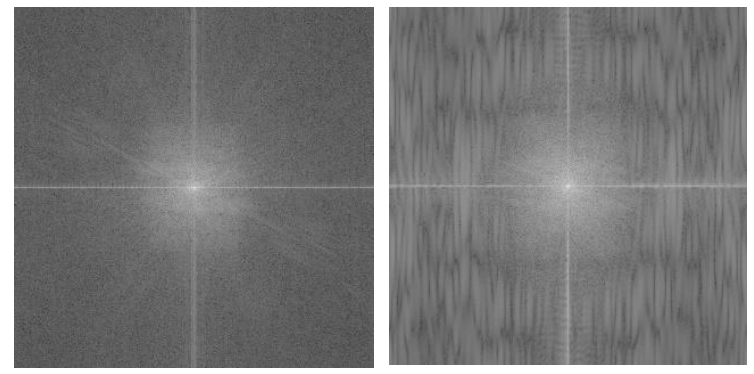

Figure 11(a): Fourier transforms of image at 9(a), (b): Fourier transform of image at 9(c).

\section{CONCLUSION}

Since we are using the secondary dataset we are analysing it using multiple pre-processing methods such as histogram, Fourier transforms and surface plot (Figure from 3 to 11) to get better synthesized image. The minimum and maximum threshold values for Local Thresholding methods (Figure 6) are approximate to similar range. Thus we can conclude that this step has been completed with an optimized and synthesized image with Hypothalamus localization. The simulation process reached to half way with the completion of initial two modules. Thus SMHB method has been partially applied and result has been evaluated for a 3D image initially and further limited data set of ten images which are divided in two different sub-categories 5 images with anatomical information and 5 images with BOLD signals with Hunger Regulation functionality.

\section{FUTURE SCOPE}

We will also applied the regression based strategy for optimized estimation of the signal mapping with zero false probability (FPDF) (Equation 2) for the mapping between sparse matrix and fMRI voxels. Wavelet transform can also be used to synthesize an fMRI image. Afterwards, successive steps will be completed and final result will be generated. A regression based statistical analysis (Gaussian model or Laplacian Model) of the sparse parameters can be performed for the fMRI data set. A supervised machine learning tool like Support vector machine (SVM) for Linear and Non Linear aspect can be for the classification method for various task and stimuli activities. A multi-voxel, multi spectral, multivariate hybrid model with supervised or reinforcement training can also be implemented in near future to find the discrepancy between fMRI signals for different physiological functions.

\section{REFERENCES}

1. Divya, Saurabh Mukherjee. (2015) "Mathematical Model to represent the role of Hypothalamus in Hunger Regulation", presented at $1^{\text {st }}$ National Seminar on "Information Technology Applications: Strategies, Issues and Challenges" at Prestige Institute of Management, Gwalior (Madhya Pradesh), ISBN- 978-93-85000-08-9.

2. Divya, Mukherjee S. (2015). Optimizing and Validating the Hypothalamic Hunger Regulation Mathematical Model. Journal of Network communications and Emerging Technologies (JNCET). 5. 203-207.

3. Abu Dayyeh, Barham \& Port, John \& Vijayvargiya, Priya \& Camilleri, Michael. (2014). Tu1798 Characterization of Hypothalamic Hunger and Satiety Signals With Pulsed Arterial Spin Labeling MRI. Gastroenterology. 146. S-845. 10.1016/S0016-5085(14)63074-2.

4. Crimi, A., Giancardo, L., Sambataro, F. et al. MultiLink Analysis: Brain Network Comparison via Sparse Connectivity Analysis. Sci Rep 9, 65 (2019) doi:10.1038/s41598-018-37300-4.

5. Rucker, P., \& Ikuta, T. (2019). Pituitary Gland Functional Connectivity and BMI. Frontiers in neuroscience, 13, 120. doi:10.3389/fnins.2019. 00120

6. Al-Zubaidi A, Mertins A, Heldmann M, Jauch-Chara K and Münte TF (2019) Machine Learning Based Classification of Resting-State fMRI Features Exemplified by Metabolic State (Hunger/Satiety). Front. Hum. Neurosci. 13:164. doi: 10.3389/fnhum.2019.00164.

7. Pinho, A., Amadon, A., Ruest, T. et al. Individual Brain Charting, a high-resolution fMRI dataset for cognitive mapping. Sci Data 5, 180105 (2018) doi:10.1038/sdata.2018.105.

8. Gauthier, C.J., Fan, A.P., BOLD signal physiology: Models and applications, NeuroImage (2018), doi: 10.1016/j.neuroimage. 2018.03.018.

9. M. K. Carroll, G. A. Cecchi, I. Rish, R. Garg, and A. R. Rao, "Prediction and interpretation of distributed neural activity with sparse models," NeuroImage, vol. 44, no. 1, pp. 112-122,2009.

10. Blanca Guillen, Jose L. Paredes, and Rubén Medina, "Detecting Activation in fMRI Data: An Approach Based on Sparse Representation of BOLD Signal," Mathematical Problems in Engineering, vol. 2018, Article ID 1730149, 15 pages, 2018. https://doi.org/10.1155/2018/1730149.

11. Smeets PA, Kroese FM, Evers C, de Ridder DT. Behav Brain Res 2013 Jul 1;248:41-5. doi: 10.1016/j.bbr.2013.03.041. Epub 2013 Apr 8. http://www.ncbi.nlm.nih.gov/pubmed/23578759.

12. Caballero-Gaudes, C., \& Reynolds, R. C. (2017). Methods for cleaning the BOLD fMRI signal. NeuroImage, 154, 128-149. doi:10.1016/j.neuroimage.2016.12.018. 
13. Al-Baddai, Saad \& Al-Subari, Karema \& Tomé, Ana \& Ludwig, Bernd \& Salas-Gonzales, Diego \& Lang, Elmar. (2016). Analysis of fMRI images with bi-dimensional empirical mode decomposition based-on Green's functions. Biomedical Signal Processing and Control, ISSN: 1746-8094, Vol: 30, Page: 53-63, Elsevier BV.

14. Abdellah, Marwan. (2015). Computational Models and Simulators of functional MRI. 10.13140/RG.2.2.24990.33600.

15. Lizarbe, B., Benitez, A., Peláez Brioso, G. A., Sánchez-Montañés, M., López-Larrubia, P., Ballesteros, P., \& Cerdán, S. (2013). Hypothalamic metabolic compartmentation during appetite regulation as revealed by magnetic resonance imaging and spectroscopy methods. Frontiers in neuroenergetics, 5, 6. doi:10.3389/fnene.2013.00006.

16. Arichi, Tomoki \& Fagiolo, Gianlorenzo \& Varela, Marta \& MelendezCalderon, Alejandro \& Allievi, Alessandro \& Merchant, Nazakat \& Tusor, Nora \& Counsell, Serena \& Burdet, Etienne \& Beckmann, Christian \& Edwards, David. (2012). Development of BOLD signal hemodynamic responses in the human brain. NeuroImage. 63. 663-73. 10.1016/j.neuroimage.2012.06.054.

17. Ildar Khalidov, Jalal Fadili, François Lazeyras, Dimitri Van De Ville, Michael Unser, Activelets: Wavelets for sparse representation of hemodynamic responses, Signal Processing, Volume 91, Issue 12, 2011, Pages 2810-2821, ISSN 0165-1684, https://doi.org/10.1016/j.sigpro. 2011. 03.008.

\section{AUTHORS PROFILE}

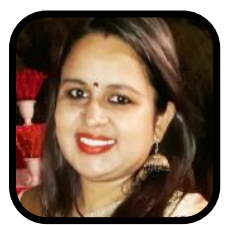

Divya is working as Assistant Professor in Department of Computer Science, Banasthali Vidyapith, Rajasthan. She is having 7 years of teaching experience. She is pursuing her research work in the field of Medical image processing, Mathematical Modeling and Computer Vision.

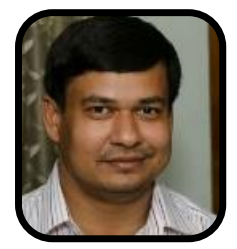

Saurabh Mukherjee, Prof. Saurabh Mukherjee is working as a Professor in the department of Computer Science, Banasthali Vidyapith, Rajasthan. He is having 20 years of teaching and research experience in Medical Image Processing, Machine Learning, Deep learning, Data science to name a few. He has guided $12 \mathrm{PhD}$ students under his credit. Currently, 06 students are pursuing $\mathrm{PhD}$ under him. 